\title{
Natural Intrinsic Geometrical Symmetries ${ }^{\star}$
}

\author{
Stefan HAESEN ${ }^{\dagger}$ and Leopold VERSTRAELEN ${ }^{\ddagger}$ \\ $\dagger$ Simon Stevin Institute for Geometry, Wilhelminaweg 1, 2042 NN Zandvoort, The Netherlands \\ E-mail: stefan.haesen@geometryinstitute.org \\ $¥$ Katholieke Universiteit Leuven, Department of Mathematics, Celestijnenlaan 200B bus 2400, \\ B-3000 Leuven, Belgium \\ E-mail: leopold.verstraelen@wis.kuleuven.be
}

Received April 08, 2009, in final form August 25, 2009; Published online September 02, 2009

doi:10.3842/SIGMA.2009.086

\begin{abstract}
A proposal is made for what could well be the most natural symmetrical Riemannian spaces which are homogeneous but not isotropic, i.e. of what could well be the most natural class of symmetrical spaces beyond the spaces of constant Riemannian curvature, that is, beyond the spaces which are homogeneous and isotropic, or, still, the spaces which satisfy the axiom of free mobility.
\end{abstract}

Key words: parallel transport; holonomy; spaces of constant curvature; pseudo-symmetry

2000 Mathematics Subject Classification: 53A55; 53B20

\section{Introduction}

Let $\left(M^{n}, g\right)$ be an arbitrary Riemannian manifold, i.e. let $M^{n}$ be any differential manifold of dimension $n$ endowed with any Riemannian metric $(0,2)$ tensor $g$. On the one hand, what follows, with the usual care, can fully be developed also for indefinite semi- or pseudo-Riemannian spaces, and, moreover, can appropriately be extended to Cartan's "generalized"-spaces. And, on the other hand, when given some extra structure on $M^{n}$, e.g. like a Kaehlerian or a Sasakian structure, what follows can accordingly be well specified too. The present presentation however will simply be restricted to some natural symmetries occurring in intrinsic proper Riemannian geometry.

A geometrical symmetry of a Riemannian space $\left(M^{n}, g\right)$ concerns the invariance of some geometrical quantity of $\left(M^{n}, g\right)$ under the performance of some transformations defined on $\left(M^{n}, g\right)$ [69]. Various types of symmetries in Riemannian geometry can thus be considered, essentially depending on the kind of quantities and of transformations in question. And, in complete analogy with such intrinsic symmetries, various types of extrinsic symmetries can be considered on submanifolds $\left(M^{n}, g\right)$ in Riemannian ambient spaces $\left(\widetilde{M}^{n+m}, \widetilde{g}\right)$. By natural geometrical symmetries, we mean symmetries in the above sense for which the quantities and the transformations involved are the most natural indeed, at least in our opinion. As more technical as well as more expository references for these intrinsic and for these extrinsic symmetries, cf. [19, 23, 28, 39, 53, 68].

\section{Transformations on Riemannian manifolds}

The transformations on Riemannian manifolds $\left(M^{n}, g\right)$ which will be considered hereafter in our speculations about symmetry will be the parallel transports fully around all the infinitesimal

\footnotetext{
*This paper is a contribution to the Special Issue "Élie Cartan and Differential Geometry". The full collection is available at http://www.emis.de/journals/SIGMA/Cartan.html
} 
co-ordinate parallelograms on these manifolds. A 4-fold motivation to devote some attention to these rather than maybe to some other kinds of transformations is the following.

(i) Essentially, when studying symmetry, one is interested in the preservation of one or other sort of measures of some geometrical "beings", cf. the "parlant" of Élie Cartan, after these beings have been transformed in one or other manner. Working in Riemannian geometry, these measures do most naturally concern measures taken by the Riemannian metric tensor g. And since this tensor may well change from point to point on a general Riemannian manifold $\left(M^{n}, g\right)$, it seems wise to restrict to transformations on these manifolds which do bring all beings living at any point $p \in M$ back to this same point $p$, so that their measurements done before and after these transformations are carried through are taken by the same measure $g(p)$. This condition seems to be the most elementary one to impose if one is willing to show respect for the metrical structure of the space $\left(M^{n}, g\right)$.

(ii) The Riemannian metrical structure $g$ is defined on a differential manifold $M^{n}$. So, the transformations to consider in the purpose of studying some basic symmetries of Riemannian manifolds $\left(M^{n}, g\right)$ should fundamentally involve the essence of what is a differential structure. An $n D$ differential structure on a space $M$ consists of an atlas of local co-ordinate systems (or patches or charts) $x^{1}, \ldots, x^{h}, \ldots, x^{k}, \ldots, x^{n}$ around its points $p$, which, in case there exists between two of such systems a non-trivial overlap, do have differential transitions of their coordinates there. So, the most elementary aspect of a differential structure on a space $M$ might well be the existence of "xy co-ordinate parallelograms $\mathcal{P}$ cornered at its points $p$ ", whereby we have renamed $x^{h}$ as $x$ and $x^{k}$ as $y$ and, keeping all other co-ordinates around a point $p=$ $\left(x^{1}, \ldots, x^{h-1}, x, x^{h+1}, \ldots, x^{k-1}, y, x^{k+1}, \ldots, x^{n}\right)$ fixed, consider the "parallelogram" $\mathcal{P}$ formed by the co-ordinate lines $x^{h}=x, x^{k}=y, x^{h}=x+\Delta x$ and $x^{k}=y+\Delta y$, for arbitrary increments $\Delta x$ and $\Delta y$ of the co-ordinates $x$ and $y$. Actually, this goes back to the essence of desCartes' systematic use of planar (oblique) $x y$ co-ordinates in his Géométrie of 1637, based there and then on the Euclidean parallel postulate.

In the following, the natural basic tangent vectors $\frac{\partial}{\partial x}(p)=\frac{\partial}{\partial x^{h}}(p)$ and $\frac{\partial}{\partial y}(p)=\frac{\partial}{\partial x^{k}}(p)$ at $p$ to the $x$ - and $y$-co-ordinate lines will be denoted by $\vec{x}$ and $\vec{y}$ respectively, and the tangent $2 D$ plane, or, still, the $2 D$ linear subspace of the tangent space $T_{p} M^{n}=\mathbb{R}^{n}$ to $M^{n}$ at $p$, which is spanned by $\vec{x}$ and $\vec{y}$ will be further denoted by $\bar{\pi}: \bar{\pi}=\vec{x} \wedge \vec{y}$. And, combining (i) and (ii) it seems no more than appropriate when showing respect respectively for the metrical and for the differential structures of general Riemannian manifolds $\left(M^{n}, g\right)$, and, being in the search of basic symmetries in Riemannian geometry, to consider some kinds of transformations starting at arbitrary points $p$ of $M$ and moving fully around co-ordinate parallelograms $\mathcal{P}$ cornered at $p$ and tangent there to arbitrary tangent 2-planes $\bar{\pi}=\vec{x} \wedge \vec{y}$.

(iii) Both in Riemann's and in Helmholtz's pioneering studies of Riemannian spaces $\left(M^{n}, g\right)$ [40, 60], with their inspirations coming (a.o.) from philosophical thoughts primordially about physical space-time and about human vision, respectively, the basic metrical geometrical structure, i.e. basically -in modern terminology- the metric tensor $g$, was essentially defined as an infinitesimal notion: $g=g_{h k} \mathrm{~d} x^{h} \mathrm{~d} x^{k}$, [whereby the components $g_{h k}$ of $g,(h, k \in\{1,2, \ldots, n\})$, are real valued functions on (open parts of) the "underlying" differential manifold, namely, $\left.g_{h k}: M \rightarrow \mathbb{R}\right]$. And this in itself essentially was possible since working on a manifold $M^{n}$ to which the infinitesimal calculus from $\mathbb{R}^{n}$ was extended via mutually compatible local charts. Accordingly, after thus reflecting a bit deeper than in $(i)$ and $(i i)$ on the infinitesimal characters of both the differential structure and the metrical structure of a Riemannian manifold, we will further on consider transformations on $\left(M^{n}, g\right)$ moving beings from any point fully around all infinitesimal co-ordinate parallelograms $\mathcal{P}$ back to $p$, i.e., in the following, $\Delta x$ and $\Delta y$ will be considered to be infinitesimal quantities. 
(iv) Finally, amongst all such transformations of beings around such parallelograms $\mathcal{P}$, the most natural ones, again with due respect for the differential structure as well as for the metrical structure of Riemannian manifolds $\left(M^{n}, g\right)$, seem well to be the corresponding parallel transports. These were introduced independently by Levi-Civita [50] and by Schouten [62], in particular aiming for truly geometrical insights in the meaning of the Riemann-Christoffel curvature tensor $R$ and of the Riemann sectional curvatures $K(p, \pi)$ of the $2 D$ sections $\pi$ of the tangent spaces $T_{p} M^{n}$ at the points $p$ of $M$. These parallel transports of vectors along curves in $\left(M^{n}, g\right)$ are conceptually equivalent with the Riemannian connection $\nabla$ which, in Koszul's approach, and as worked out by Nomizu, according to the fundamental lemma of Riemannian geometry, is the unique way to associate a vector field $\nabla_{X} Y$ with given vector fields $X$ and $Y$ on $\left(M^{n}, g\right)$ following the derivation rules of linearity and of Leibniz and being compatible with the differential structure and with the metrical structure of $\left(M^{n}, g\right)$, i.e., being symmetrical (that is, the commutator $\nabla_{X} Y-\nabla_{Y} X$ which is defined for two such vector fields $X$ and $Y$ in terms of this connection coincides with the commutator, or Lie bracket, $[X, Y]=X Y-Y X$, which is defined for these vector fields by the differential structure) and being metrical (that is, $Z[g(X, Y)]-g\left(\nabla_{Z} X, Y\right)-g\left(X, \nabla_{Z} Y\right)=0$, or, put otherwise, $\nabla g=0$, or still, the scalar product of vectors does not change under their parallel transports along curves).

As is well known, and goes back to Schouten [62], one has the following holonomy property: after parallel transport of a vector $\vec{z} \in T_{p} M^{n}$ fully around a co-ordinate parallelogram $\mathcal{P}$ cornered at $p$ and tangent there to $\bar{\pi}=\vec{x} \wedge \vec{y}$, results the vector

$$
\vec{z}_{\bar{\pi}}^{\star}=\vec{z}+[R(\vec{x}, \vec{y}) \vec{z}] \Delta x \Delta y+\mathcal{O}^{>2}(\Delta x, \Delta y),
$$

whereby $R(X, Y): T M \rightarrow T M$ is the curvature operator, i.e. $R(X, Y) Z:=\left(\nabla_{X} \nabla_{Y}-\nabla_{Y} \nabla_{X}-\right.$ $\left.\nabla_{[X, Y]}\right) Z$, or still, whereby $R: T M \times T M \times T M \rightarrow T M:(X, Y, Z) \mapsto R(X, Y) Z$ is the $(1,3)$ Riemann-Christoffel curvature tensor. Thus $R(\vec{x}, \vec{y}) \vec{z}$ measures the second order change of a vector $\vec{z} \in T_{p} M^{n}$ after parallel transport around an infinitesimal co-ordinate parallelogram $\mathcal{P}$. Or, by taking into account that parallel transports are isometries, and so, in particular, do not change the lengths of vectors, one has the following.

Proposition 1 (Schouten). The curvature operators of Riemannian manifolds measure the changes of directions at points under parallel transports fully around the infinitesimal parallelograms cornered at these points.

Actually, the above holonomy property of Schouten during the last decades is often used as the definition of the $(1,3)$ curvature tensor $R$. And, stated as the symmetry property of preserving directions for the class of transformations under consideration, one has the following.

Theorem 1 (Schouten). The locally Euclidean (or locally flat) Riemannian manifolds (the spaces $\left(M^{n}, g\right)$ for which $\left.R \equiv 0\right)$, are precisely the Riemannian manifolds for which all directions are invariant under their parallel transports fully around all infinitesimal co-ordinate parallelograms.

Remark 1. Actually, it was Cartan who very appropriately introduced the term "holonomy" by combining the Greek "holos" and "nomos".

\section{Geometrical meaning of the metrical endomorphism}

The natural metrical endomorphism $X \wedge_{g} Y: T M \rightarrow T M$ associated with two vector fields $X$ and $Y$ on a Riemannian manifold $\left(M^{n}, g\right)$ is defined by $\left(X \wedge_{g} Y\right) Z:=g(Y, Z) X-g(X, Z) Y$. Let $\vec{x}$ and $\vec{y}$ be orthonormal vectors at $p$, and let $\vec{z}=\vec{z}_{\bar{\pi}}+\vec{z}_{\bar{\pi}^{\perp}}$ be the canonical orthogonal 
decomposition of any vector $\vec{z}$ at $p$ in its components in $\bar{\pi}=\vec{x} \wedge \vec{y}$ and in the orthogonal complement $\bar{\pi}^{\perp}$ of $\bar{\pi}$ in $T_{p} M^{n}=\mathbb{R}^{n}$. Now, we rotate $\vec{z}_{\bar{\pi}}$ around $p$ in the plane $\bar{\pi}$ over an infinitesimal angle $\Delta \varphi$, thus obtaining a vector $\left(\vec{z}_{\bar{\pi}}\right)_{\Delta \varphi}$, and define the vector $\vec{z}_{\bar{\pi}}^{\wedge}:=\left(\vec{z}_{\bar{\pi}}\right)_{\Delta \varphi}+\vec{z}_{\bar{\pi}^{\perp}}$. The procedure going from $\vec{z}$ to $\vec{z}_{\bar{\pi}}^{\wedge}$ is called the rotation of $\vec{z}$ at $p$ with respect to the plane $\bar{\pi}$ over an angle $\Delta \varphi$, and one has the following:

$$
\vec{z}_{\bar{\pi}}^{\wedge}=\vec{z}+\left[\left(\vec{x} \wedge_{g} \vec{y}\right) \vec{z}\right] \Delta \varphi+\mathcal{O}^{>1}(\Delta \varphi) .
$$

Thus the vector $\left(\vec{x} \wedge_{g} \vec{y}\right) \vec{z}$ measures the first order change of the vector $\vec{z}$ after an infinitesimal rotation of $\vec{z}$ at $p$ with respect to the plane $\bar{\pi}=\vec{x} \wedge \vec{y}$, or, formulated more loosely, we have the following.

Proposition 2 ([39]). The natural metrical endomorphisms $\wedge_{g}$ of Riemannian manifolds measure the changes of directions at points under infinitesimal rotations with respect to $2 D$ planes at these points.

\section{The sectional curvatures of Riemann}

The $(0,4)$ Riemann-Christoffel curvature tensor $R$ of $\left(M^{n}, g\right)$ is related to the $(1,3)$ tensor $R$ by $R(X, Y, Z, W)=g(R(X, Y) Z, W)$. The simplest $(0,4)$ tensor which is canonically determined on $\left(M^{n}, g\right)$ and which has the same algebraic symmetry properties as $R$ likely is the tensor $G(X, Y, Z, W):=g\left(\left(X \wedge_{g} Y\right) Z, W\right)$, or, still, in terms of the Nomizu-Kulkarni product of $(0,2)$ tensors: $G=\frac{1}{2} g \wedge g$, i.e., basically $G$ is the Nomizu-Kulkarni square of the metric $(0,2)$ tensor $g$.

Let $\vec{v}$ and $\vec{w}$ be any pair of linearly independent vectors to $M$ at a point $p$ spanning there a $2 D$ plane $\pi=\vec{v} \wedge \vec{w} \subset T_{p} M^{n}$. Then, the sectional curvature $K(p, \pi):=R(\vec{v}, \vec{w}, \vec{w}, \vec{v}) / G(\vec{v}, \vec{w}, \vec{w}, \vec{v})$ of $M^{n}$ at $p$ for $\pi$, (the definition is independent of the choice of basis $\vec{v}, \vec{w}$ for $\pi$ ), i.e., cf. Riemann, the Gauss curvature at $p$ of the 2-dimensional surface $G_{\pi}^{2} \subset M^{n}$ consisting locally of all the geodesics of $\left(M^{n}, g\right)$ passing through $p$ and whose velocity vectors at $p$ belong to $\pi$ (such that $T_{p} G_{\pi}^{2}=\pi$ ), can be thought of as a kind of nomalisation of the curvature operator by the natural metrical endomorphism, or, still, of the parallel transport of directions in $M$ at $p$ around co-ordinate parallelograms cornered at $p$ by the rotations of directions in $M$ at $p$ with respect to planes in $T_{p} M$. For any orthonormal basis $\vec{v}, \vec{w}$ of $\pi$, of course: $K(p, \pi)=R(\vec{v}, \vec{w}, \vec{w}, \vec{v})$.

Levi-Civita [50] gave a geometrical interpretation of the sectional curvatures $K(p, \pi)$ in terms of his parallelogramoids, of which we next consider the special case of his squaroids. Let $\vec{v}$ and $\vec{w}$ be any orthonormal basis of a tangent 2-plane $\pi$ of $\left(M^{n}, g\right)$ at $p$. Consider the geodesic $\alpha$ through $p=\alpha(0)$ with velocity $\alpha^{\prime}(0)=\vec{w}$, and on it then localise a point $q=\alpha(\varepsilon)$ at an infinitesimal distance $\varepsilon$ from $p$. Then move $\vec{v}$ parallel along $\alpha$ from $p$ to $q$, thus obtaining at $q$ the vector $\vec{v}_{\alpha}^{\star}$. Through $p$ and $q$, further, we consider the geodesics $\beta$ and $\gamma, \beta(0)=p$ and $\gamma(0)=q$, with respective velocities $\beta^{\prime}(0)=\vec{v}$ and $\gamma^{\prime}(0)=\vec{v}_{\alpha}^{\star}$. On these geodesics we then localise the points $\bar{p}=\beta(\varepsilon)$ and $\bar{q}=\gamma(\varepsilon)$ at distances $\varepsilon$ from $p$ and $q$, respectively. Finally, a Levi-Civita squaroid $p q \bar{q} \bar{p}$ is completed by joining $\bar{p}$ and $\bar{q}$ by a geodesic $\delta$. In general, the geodesic distance between $\bar{p}$ and $\bar{q}$ will be different from $\varepsilon$ and, denoting this distance by $\varepsilon^{\prime}$, one has the following.

Theorem 2 (Levi-Civita). In first order approximation: $K(p, \pi)=\left(\varepsilon^{2}-\varepsilon^{\prime 2}\right) / \varepsilon^{4}$.

As references, and, in some cases, as sources for more precise statements for the following classical results, see e.g. [43, 44, 49, 71].

Theorem 3 (Cartan). The knowledge of the $(0,4)$ curvature tensor $R$ is equivalent to the knowledge of all sectional curvatures $K(p, \pi)$ of Riemann.

Theorem 1 can be reformulated as follows. 
Theorem 4 (Schouten). The Riemannian manifolds with constantly vanishing sectional curvature of Riemann, $K(p, \pi) \equiv 0$, are precisely the spaces for which all directions are invariant under their parallel transports fully around all infinitesimal co-ordinate parallelograms.

A Riemannian manifold $\left(M^{n}, g\right)$ is said to be a space of constant curvature $K$ or a real space form $M^{n}(c)$ when for all points $p$ and for all planar sections $\pi \subset T_{p} M$ one has $K(p, \pi)=K=$ $c \in \mathbb{R}$.

Theorem 5. A Riemannian manifold $\left(M^{n}, g\right), n \geq 2$, is a real space form if and only if $R=c G$, $c \in \mathbb{R}$.

Theorem 6 (Schur). A Riemannian manifold $\left(M^{n}, g\right), n \geq 3$, for which all sectional curvatures $K$ are isotropic, i.e., a Riemannian manifold for which for every one of its points $p$ the curvatures $K(p, \pi)$ are the same for all possible planar sections $\pi \subset T_{p} M$, has constant sectional curvatures $K(p, \pi)$, or, still, their sectional curvatures $K$ further are also independent of the points $p$.

Theorem 7 (Riemann). Any two nD Riemannian manifolds with the same constant sectional curvatures are locally isometric; actually, in local co-ordinates, the metrical fundamental form of a real space form of curvature $c$ can be expressed as $\mathrm{d} s^{2}=\left\{1+\frac{c}{4} \sum_{j}\left(x^{j}\right)^{2}\right\}^{-2} \sum_{i}\left(\mathrm{~d} x^{i}\right)^{2}$.

Theorem 8 (Killing, Hopf). The Euclidean spaces $\mathbb{E}^{n}(c=0)$ and the classical non-Euclidean geometries, of elliptic type on the spheres $\mathbb{S}^{n}(c>0)$ and of hyperbolic type on the spaces $\mathbb{H}^{n}$ $(c<0)$, are the model spaces of the real space forms $M^{n}(c), n \geq 2$.

Moreover, we recall that the real space forms may well be considered to be the utmost possible symmetrical Riemannian spaces, in the sense that they are homogeneous (i.e. they "behave" the same at all of their points $p$ ) and that they are isotropic (i.e. they "behave" the same in all directions). Both conditions together are a way to express that the isometry groups of the real space forms are of maximal dimension possible amongst all Riemannian manifolds of the same dimensions, or, still, that we have the following.

Theorem 9 (Riemann, Helmholtz, Lie, Klein, Tits). A Riemannian manifold $\left(M^{n}, g\right)$ is a real space form $M^{n}(c)$ if and only if it satisfies the axiom of free mobility.

Finally, we recall also the fundamental following result (for a new recent proof of which we refer to Matveev [54]), which in some sense unifies the Euclidean and the classical non-Euclidean geometries from a projective point of view.

Theorem 10 (Beltrami). The real space forms constitute the projective class of the locally Euclidean spaces, or, still, by applying geodesic transformations to locally Euclidean spaces one obtains spaces of constant curvature and the class of the spaces of constant curvature is closed under geodesic transformations.

\section{Geometrical meaning of semi-symmetry}

The curvatures $R$ and $K$ of a Riemannian manifold $\left(M^{n}, g\right)$ are its main metrical invariants [3, 4]. They essentially involve the second order derivatives, as those are determined by the differential structure of the manifold $M^{n}$, of the metrical structure of the Riemannian manifold, i.e. of $g$.

Let $\vec{v}$ and $\vec{w}$ be any orthonormal vectors at any point $p$ in a Riemannian manifold, and let $\mathcal{P}$ be any infinitesimal co-ordinate parallelogram cornered at $p$ as before, i.e. in particular being tangent at $p$ to the plane $\bar{\pi}=\vec{x} \wedge \vec{y}$. By parallel transport of $\vec{v}$ and $\vec{w}$ around $\mathcal{P}$ then result the orthonormal vectors $\vec{v}^{\star}=\vec{v}+[R(\vec{x}, \vec{y}) \vec{v}] \Delta x \Delta y+\mathcal{O}^{>2}(\Delta x, \Delta y)$ and $\vec{w}^{\star}=\vec{w}+[R(\vec{x}, \vec{y}) \vec{w}] \Delta x \Delta y+$ 
$\mathcal{O}^{>2}(\Delta x, \Delta y)$, such that the plane $\pi=\vec{v} \wedge \vec{w}$ at $p$ is accordingly parallel transported around $\mathcal{P}$ into the plane $\pi^{\star}=\vec{v}^{\star} \wedge \vec{w}^{\star}$ at $p$. Hence, $K\left(p, \pi^{\star}\right)=R\left(\vec{v}^{\star}, \vec{w}^{\star}, \vec{w}^{\star}, \vec{v}^{\star}\right)=R(\vec{v}, \vec{w}, \vec{w}, \vec{v})+$ $[R(R(\vec{x}, \vec{y}) \vec{v}, \vec{w}, \vec{w}, \vec{v})+R(\vec{v}, R(\vec{x}, \vec{y}) \vec{w}, \vec{w}, \vec{v})+R(\vec{v}, \vec{w}, R(\vec{x}, \vec{y}) \vec{w}, \vec{v})+R(\vec{v}, \vec{w}, \vec{w}, R(\vec{x}, \vec{y}) \vec{v})] \Delta x \Delta y+$ $\mathcal{O}^{>2}(\Delta x, \Delta y)=K(p, \pi)-[(R \cdot R)(\vec{v}, \vec{w}, \vec{w}, \vec{v} ; \vec{x}, \vec{y})] \Delta x \Delta y+\mathcal{O}^{>2}(\Delta x, \Delta y)$, whereby $R \cdot R$ denotes the $(0,6)$ tensor which is obtained by the actions of the curvature operators $R(X, Y)$ as derivations on the $(0,4)$ Riemann-Christoffel curvature tensor.

Proposition 3 ([39]). The curvature tensor $R \cdot R$ measures the changes of the sectional curvatures $K(p, \pi)$ of a Riemannian manifold $\left(M^{n}, g\right)$ for all planes $\pi$ at all points $p$ under the parallel transports of these planes $\pi$ fully around all infinitesimal parallelograms $\mathcal{P}$ cornered at $p$ and tangent there to all planes $\bar{\pi}$.

The Riemannian manifolds $\left(M^{n}, g\right)$ for which $R \cdot R=0$ are called semi-symmetric or Szabó symmetric. These spaces were classified in general by Szabó [63, 64]; for more specific information on some particular cases, see also Boeckx [5], Kowalski [46] and Lumiste [53].

Theorem 11 ([39]). The semi-symmetric Riemannian spaces are precisely the Riemannian manifolds for which, up to second order, all the sectional curvatures are invariant under their parallel transports fully around all infinitesimal co-ordinate parallelograms.

Theorem 12 (Szabó). Let $\left(M^{n}, g\right)$ be a semi-symmetric Riemannian manifold of dimension $n>2$. Then there is an everywhere dense open subset $U \subset M$ such that around every point $p \in U$ the space $M$ is locally isometric to a direct product of an open part of a Euclidean space and some infinitesimally irreducible simple semi-symmetric leaves $N$ which are: $(i)$ locally symmetric if $\nu_{p}=0$ and $u_{p}>2$; (ii) locally isometric to a Euclidean, an elliptical or a hyperbolic cone if $\nu_{p}=1$ and $u_{p}>2$; (iii) locally isometric to a Kaehlerian cone if $\nu_{p}=2$ and $u_{p}>2$; and (iv) locally isometric to a space foliated by Euclidean 2-codimensional leaves if $\nu_{p}=n-2$ and $u_{p}=2$, whereby $\nu_{p}$ and $u_{p}$ respectively are the indices of nullity and of conullity of $\left(M^{n}, g\right)$ at $p$.

The curvature tensor $R$ of a 2-dimensional Riemannian manifold $\left(M^{2}, g\right)$ essentially reduces to the Gauss curvature function $K: M^{2} \rightarrow \mathbb{R}$ and so, by the Theorem of Schwarz-Young, every $2 D$ Riemannian space is automatically semi-symmetric. The curvature condition $R \cdot R=0$ first appeared in the studies concerning locally symmetric spaces by P.A. Shirokov and by É. Cartan, namely as integrability condition of the curvature condition $\nabla R=0$. For more information about the origins of the notions of semi- and of pseudo-symmetry, in particular concerning the mayor influences in these contexts of the articles [56] and [11] of Nomizu and Chen, respectively, see [53] and [68]. At this stage, we will confine to the comment that whereas the curvature condition to be locally symmetric or Cartan symmetric $(\nabla R \equiv 0)$ essentially involve the 3rd order derivatives of the metric of a Riemannian space $\left(M^{n}, g\right)$, the curvature condition to be semisymmetric or Szabó symmetric $(R \cdot R \equiv 0)$ again essentially involves the 2nd order derivatives of the metric $g$.

Proposition 4 ([39]). The $(0,6)$ tensor

$$
\begin{aligned}
(R \cdot R) & \left(X_{1}, X_{2}, X_{3}, X_{4} ; X, Y\right):=(R(X, Y) \cdot R)\left(X_{1}, X_{2}, X_{3}, X_{4}\right) \\
= & -R\left(R(X, Y) X_{1}, X_{2}, X_{3}, X_{4}\right)-R\left(X_{1}, R(X, Y) X_{2}, X_{3}, X_{4}\right) \\
& -R\left(X_{1}, X_{2}, R(X, Y) X_{3}, X_{4}\right)-R\left(X_{1}, X_{2}, X_{3}, R(X, Y) X_{4}\right)
\end{aligned}
$$

has the following algebraic symmetry properties:

a) $(R \cdot R)\left(X_{1}, X_{2}, X_{3}, X_{4} ; X, Y\right)=-(R \cdot R)\left(X_{2}, X_{1}, X_{3}, X_{4} ; X, Y\right)$

$$
=(R \cdot R)\left(X_{3}, X_{4}, X_{1}, X_{2} ; X, Y\right) \text {, }
$$


b) $(R \cdot R)\left(X_{1}, X_{2}, X_{3}, X_{4} ; X, Y\right)+(R \cdot R)\left(X_{1}, X_{3}, X_{4}, X_{2} ; X, Y\right)$

$+(R \cdot R)\left(X_{1}, X_{4}, X_{2}, X_{3} ; X, Y\right)=0$

c) $(R \cdot R)\left(X_{1}, X_{2}, X_{3}, X_{4} ; X, Y\right)=-(R \cdot R)\left(X_{1}, X_{2}, X_{3}, X_{4} ; Y, X\right)$,

d) $(R \cdot R)\left(X_{1}, X_{2}, X_{3}, X_{4} ; X, Y\right)+(R \cdot R)\left(X_{3}, X_{4}, X, Y ; X_{1}, X_{2}\right)$

$+(R \cdot R)\left(X, Y, X_{1}, X_{2} ; X_{3}, X_{4}\right)=0$.

\section{The sectional curvatures of Deszcz}

The simplest non-trivial $(0,6)$ tensor which is canonically determined on $\left(M^{n}, g\right)$ and which has the same algebraic symmetry properties as $R \cdot R$ likely is the Tachibana tensor

$$
\begin{aligned}
& \left(\wedge_{g} \cdot R\right)\left(X_{1}, X_{2}, X_{3}, X_{4} ; X, Y\right):=\left(\left(X \wedge_{g} Y\right) \cdot R\right)\left(X_{1}, X_{2}, X_{3}, X_{4}\right) \\
& =-R\left(\left(X \wedge_{g} Y\right) X_{1}, X_{2}, X_{3}, X_{4}\right)-R\left(X_{1},\left(X \wedge_{g} Y\right) X_{2}, X_{3}, X_{4}\right) \\
& \quad-R\left(X_{1}, X_{2},\left(X \wedge_{g} Y\right) X_{3}, X_{4}\right)-R\left(X_{1}, X_{2}, X_{3},\left(X \wedge_{g} Y\right) X_{4}\right),
\end{aligned}
$$

i.e. is the $(0,6)$ tensor resulting from the actions as derivations of the metrical endomorphisms $X \wedge_{g} Y$ on the $(0,4)$ curvature tensor $R$.

Theorem 13 (cf. [32]). The Tachibana tensor of a Riemannian manifold $\left(M^{n}, g\right), n \geq 3$, vanishes identically, $\wedge_{g} \cdot R \equiv 0$, if and only if $\left(M^{n}, g\right)$ is a real space form $M^{n}(c)$.

In view of the geometrical interpretation of the metrical endomorphism $\vec{x} \wedge_{g} \vec{y}$ given above in terms of infinitesimal rotations at $p \in M^{n}$ with respect to planes $\bar{\pi}=\vec{x} \wedge \vec{y}$, in particular, for orthonormal vectors $\vec{v}$ and $\vec{w}$ at $p$ one has $\vec{v}_{\bar{\pi}}^{\wedge}=\vec{v}+\left[\left(\vec{x} \wedge_{g} \vec{y}\right) \vec{v}\right] \Delta \varphi+\mathcal{O}^{>1}(\Delta \varphi)$ and $\vec{w}_{\bar{\pi}}^{\wedge}=\vec{w}+\left[\left(\vec{x} \wedge_{g}\right.\right.$ $\vec{y}) \vec{w}] \Delta \varphi+\mathcal{O}^{>1}(\Delta \varphi)$, such that the plane $\pi=\vec{v} \wedge \vec{w}$ at $p$ is accordingly rotated at $p$ with respect to the plane $\bar{\pi}=\vec{x} \wedge \vec{y}$ into the plane $\pi^{\wedge}=\vec{v}_{\bar{\pi}}^{\wedge} \wedge \vec{w}_{\bar{\pi}}^{\wedge}$ at $p$. And hence, completely analogously to a previous calculation: $K\left(p, \pi^{\wedge}\right)=K(p, \pi)+\left[\left(\wedge_{g} \cdot R\right)(\vec{v}, \vec{w}, \vec{w}, \vec{v} ; \vec{x}, \vec{y})\right] \Delta \varphi+\mathcal{O}^{>1}(\Delta \varphi)$.

Proposition 5 ([39]). The Tachibana tensor $\wedge_{g} \cdot R$ measures the changes of the sectional curvatures $K(p, \pi)$ of a Riemannian manifold $\left(M^{n}, g\right)$ for all planes $\pi$ at all points $p$ under the infinitesimal rotations of these planes $\pi$ at $p$ with respect to all planes $\bar{\pi}$ at $p$.

Theorem 14 ([39]). The real space forms are precisely the Riemannian manifolds for which, up to first order, all the sectional curvatures are invariant under their infinitesimal rotations at all points with respect to all planes.

Next, in analogy with the kind of normalisation of the changes of directions under parallel transports around parallelograms $\mathcal{P}$ cornered at $p$ by the changes of directions under rotations at $p$ with respect to planes $\bar{\pi}$ at $p$, which results in the notion of the sectional curvatures $K(p, \pi)$ of Riemann, we introduce the notion of the double sectional curvatures or the sectional curvatures $L(p, \pi, \bar{\pi})$ of Deszcz on Riemannian manifolds $\left(M^{n}, g\right)$ by now considering the changes of the sectional curvatures $K(p, \pi)$ instead of the previous changes of directions. In order to do so, for a trivial technical reason, we can meaningly in this respect only consider planes $\pi=\vec{v} \wedge \vec{w}$ and $\bar{\pi}=\vec{x} \wedge \vec{y}$ at $p$ such that $\left(\wedge_{g} \cdot R\right)(\vec{v}, \vec{w}, \vec{w}, \vec{v} ; \vec{x}, \vec{y}) \neq 0$, in which case $\pi$ is said to be curvature dependent on $\bar{\pi}$. For such pairs of planes $\pi$ and $\bar{\pi}$ then, their sectional curvature of Deszcz is defined by $L(p, \pi, \vec{\pi})=(R \cdot R)(\vec{v}, \vec{w}, \vec{w}, \vec{v} ; \vec{x}, \vec{y}) /\left(\wedge_{g} \cdot R\right)(\vec{v}, \vec{w}, \vec{w}, \vec{v} ; \vec{x}, \vec{y})$. This definition is independent of the choices of bases for the planes $\pi$ and $\bar{\pi}$. Similar to Theorem 3 of Cartan, we have the following result and its consequences.

Theorem 15 ([39]). The knowledge of the $(0,6)$ curvature tensor $R \cdot R$ is equivalent to the knowledge of all sectional curvatures $L(p, \pi, \bar{\pi})$ of Deszcz. 
Theorem 16 ([39]). A Riemannian manifold has constantly vanishing sectional curvatures of Deszcz, $L(p, \pi, \bar{\pi}) \equiv 0$, if and only if it is semi-symmetric, i.e. if, up to second order, all its sectional curvatures $K(p, \pi)$ of Riemann are invariant under the parallel transport fully around all infinitesimal co-ordinate parallelograms cornered at $p$ and tangent there to $\bar{\pi}$.

In terms of the squaroids of Levi-Civita, a geometrical interpretation of the sectional curvatures $L(p, \pi, \bar{\pi})$ of Deszcz can be given as follows. Consider a squaroid $\mathcal{S}$ of sides $\varepsilon, \varepsilon^{\prime}$, which is constructed upon orthonormal vectors $\vec{v}$ and $\vec{w}$ at a point $p$. Then, let $\vec{v}^{\star}, \vec{w}^{\star}$ and, respectively $\vec{v} \bar{\pi}, \vec{w}_{\bar{\pi}}^{\wedge}$ denote the orthonormal vectors at $p$ which result, respectively, from the parallel translation of $\vec{v}, \vec{w}$ around an infinitesimal co-ordinate parallelogram $\mathcal{P}$ and from an associated infinitesimal rotation of $\vec{v}, \vec{w}$ at $p$ with respect to a plane $\bar{\pi}=\vec{x} \wedge \vec{y}$, whereby this association means that the infinitesimal orders of the increments of the co-ordinates or angles concerned do correspond, namely that $\Delta \varphi=\Delta x \Delta y$. The sides of the squaroids $\mathcal{S}^{\star}$ and $\mathcal{S}^{\wedge}$ constructed respectively upon the vectors $\vec{v}^{\star}, \vec{w}^{\star}$ and $\vec{v}_{\bar{\pi}}^{\wedge}, \vec{w}_{\bar{\pi}}^{\wedge}$ will be denoted respectively by $\varepsilon, \varepsilon^{\star \prime}$ and by $\varepsilon, \varepsilon^{\wedge \prime}$.

Theorem 17 ([41]). In first order approximation: $L(p, \pi, \bar{\pi})=\left[\left(\varepsilon^{\star \prime}\right)^{2}-\left(\varepsilon^{\prime}\right)^{2}\right] /\left[\left(\varepsilon^{\wedge \prime}\right)^{2}-\left(\varepsilon^{\prime}\right)^{2}\right]$.

\section{Geometrical meaning of pseudo-symmetry}

A Riemannian manifold $\left(M^{n}, g\right),(n \geq 3)$, is said to be pseudo-symmetric in the sense of Deszcz or is called Deszcz symmetric if, for some function $L_{R}: M \rightarrow \mathbb{R}, R \cdot R=L_{R} \wedge_{g} \cdot R$. We observe that there does not hold a strict analog of the Theorem of Schur (concerning the curvature function $K: M \rightarrow \mathbb{R}$ in $R=K G$ ) in the case of pseudo-symmetric manifolds; see [19, 22, 37] for examples for which the function $L_{R}$ is not constant. Following Kowalski and Sekizawa [47, 48], the pseudo-symmetric spaces with constant function $L_{R}$ are said to be pseudo-symmetric of constant type.

Theorem 18 ([39]). A Riemannian manifold $\left(M^{n}, g\right),(n \geq 3)$, is Deszcz symmetric if and only if its sectional curvature of Deszcz $L(p, \pi, \bar{\pi})$ is isotropic, i.e., if $L(p, \pi, \bar{\pi})$ is independent of the planes $\pi$ and $\bar{\pi}$, or, still, if the double sectional curvature function $L(p, \pi, \bar{\pi})$ actually is a function $L=L_{R}: M \rightarrow \mathbb{R}$.

The condition for a Riemannian manifold to be pseudo-symmetric, a terminology which first appeared, as far as we know, in [20], did occur in Grycak's investigations of semi-symmetric warped products [35], as well as in the study of geodesic mappings on semi-symmetric spaces, amongst others by Sinyukov, Mikeš and Venzi [55, 67]. From these studies and from the article [15] of Defever and Deszcz we quote the following theorem, to be considered in some sense in analogy with Theorem 10 of Beltrami.

Theorem 19 (Sinyukov, Mikeš, Venzi, Defever and Deszcz). If a semi-symmetric Riemannian space admits a geodesic transformation onto some other Riemannian manifold, then this latter manifold must itself be pseudo-symmetric, and, if a pseudo-symmetric Riemannian space admits a geodesic transformation onto some other Riemannian manifold, then this latter manifold must itself also be pseudo-symmetric.

In any case, in the late 19seventies and early 19eighties the relevance of the intrinsic pseudosymmetry became more clear mainly by some studies concerning the geometry of submanifolds, notably starting with the studies, in particular by Deszcz, on extrinsic spheres in semi-symmetric spaces, which extended studies on totally umbilical submanifolds in Cartan symmetric spaces by Chen [11] and Olszak [57]. 


\section{Further types of pseudo-symmetry curvature conditions}

In full analogy with the above mentioned studies concerning the parallel transport around infinitesimal co-ordinate parallelograms of the Riemann curvatures leading to the notions of pseudo-symmetry of Riemannian manifolds and of the sectional curvatures of Deszcz, the consideration of the Weyl conformal curvatures and of the Ricci curvatures instead lead to the notions of Weyl and Ricci pseudo-symmetry and of the Weyl and Ricci curvatures of Deszcz [41, 42]. For some insight into the relationships between these various types of pseudo-symmetry curvature conditions, see e.g. [23, 38].

The following seems to be a further natural curvature condition of a similar kind, $C \cdot C=$ $L_{C} \wedge_{g} \cdot C$, whereby $C$ denotes the $(0,4)$ Weyl conformal curvature tensor as well as the corresponding curvature operator and $L_{C}$ is a real function on the manifold $M^{n}$, which condition, in contrast to the above conditions, is invariant under conformal transformations of the metric $g$. Spaces $\left(M^{n}, g\right)$ satisfying this condition are said to have a pseudo-symmetric Weyl tensor C.

In connection with further structures on Riemannian spaces, the above mentioned studies can be adapted accordingly. For instance, on a Kaehlerian manifold $\left(M^{n}, g, J\right)$ it seems most natural to focus in particular on the invariance of the holomorphic sectional curvatures under their parallel transport around infinitesimal holomorphic co-ordinate parallelograms, etc.; the results on several of such specialisations of the above general Riemannian pseudo-symmetry curvature conditions are being considered at present.

\section{Some examples of pseudo-symmetric spaces}

Of course, according to Theorem 12, starting from Szabó's classification of the semi-symmetric Riemannian spaces, by applying, eventually iteratively, geodesic transformations, one always obtains Riemannian manifolds $\left(M^{n}, g\right)$ which are pseudo-symmetric in the sense of Deszcz.

On the other hand, let $M^{n}$ be a hypersurface in a Euclidean space $\mathbb{E}^{n+1}, n \geq 3$. Amongst the simplest possible forms of the shape operator of $M^{n}$ in $\mathbb{E}^{n+1}$, one has those whereby the principal curvatures at every point are $(1):(0,0, \ldots, 0) ;(2):(\lambda, \lambda, \ldots, \lambda), \lambda \neq 0 ;(3):(\lambda, 0, \ldots,, 0)$, $\lambda \neq 0 ;(4):(\lambda, \ldots, \lambda, 0, \ldots, 0), \lambda \neq 0$ and $\lambda$ appearing more than once; $(5):(\lambda, \mu, 0, \ldots, 0)$, $\lambda \neq 0 \neq \mu$ and $\lambda \neq \mu$; (6): $(\lambda, \mu, \ldots, \mu), \lambda \neq 0 \neq \mu$ and $\lambda \neq \mu ;(7):(\lambda, \ldots, \lambda, \mu, \ldots, \mu)$, $\lambda \neq 0 \neq \mu$ and $\lambda \neq \mu$ and both $\lambda$ and $\mu$ appearing more than once. Then there are the following correspondences: $M^{n} \subset \mathbb{E}^{n+1}$ is totally geodesic in case (1); (non-totally geodesic) totally umbilical in case (2); (non-totally geodesic) cylindrical in case (3); the cases (1), (2) and (3) together cover the hypersurfaces of constant sectional curvature, i.e. the $M^{n}$ in $\mathbb{E}^{n+1}$ which are real space forms (which for these hypersurfaces is equivalent to being Einstein) and cases (1) and (3) deal with the locally flat hypersurfaces; semi-symmetric hypersurfaces which are not real space forms concern the cases (4) and (5), so that the semi-symmetric hypersurfaces $M^{n}$ of $\mathbb{E}^{n+1}$ correspond to (1), (2), (3), (4) and (5), as shown by Nomizu; conformally flat $M^{n}$, for $n>3$, which are not of constant curvature correspond to case (6); and the intrinsic pseudosymmetric $M^{n}$ in $\mathbb{E}^{n+1}$ correspond to (1), (2), (3), (4), (5), (6) and (7). So, a hypersurface $M^{n}$ in $\mathbb{E}^{n+1}$ is a non semi-symmetric, intrinsically pseudo-symmetric Riemannian manifold if and only if it has exactly two non-zero principal curvatures $\lambda$ and $\mu$, and then its double sectional curvature is given by $L=\lambda \mu$. A few further notes might be in place here. (i) A hypersurface $M^{n}$ in $\widetilde{M}^{n+1}$ is said to be quasi-umbilical when it has a principal curvature of multiplicity $\geq n-1$, and, in conformally flat ambient spaces, as shown by Cartan and Schouten, this is equivalent to $M^{n}$ being itself conformally flat, whenever $n>3$. (ii) We recall that a hypersurface $M^{n}$ in $\widetilde{M}^{n+1}(c)$ is called a cyclide of Dupin if it has, at every point, exactly two distinct principal curvatures which are both constant along the corresponding principal tangent subbundles, see e.g. $[9,10]$. (iii) A hypersurface $M^{n}$ in $\widetilde{M}^{n+1}$ is said to be 2-quasi-umbilical when it has 
a principal curvature of multiplicity $\geq n-2$, and, in particular, a hypersurface $M^{n}$ in Euclidean space $\mathbb{E}^{n+1}$, with dimension $n>3$, has a pseudo-symmetric Weyl tensor if and only if $M$ is pseudo-symmetric or its principal curvatures are given by $(\lambda, \mu, \nu, \ldots, \nu)$ whereby $\lambda \neq \mu \neq \nu \neq \lambda$.

A submanifold $M^{n}$ of a Riemannian manifold $\widetilde{M}^{n+m}$ is said to be $\vec{H}$-parallel if $R^{\perp} \cdot \vec{H}=\overrightarrow{0}$, i.e. if, up to second order approximation, the mean curvature vector $\vec{H}$ remains invariant under the $\nabla^{\perp}$ parallel transport along $M$ in $\widetilde{M}$ completely around infinitesimal co-ordinate parallelograms $\mathcal{P}$ in $M$ [28]. All pseudo-parallel submanifolds $M$ in $\widetilde{M}$ are $\vec{H}$-parallel, and, every extrinsically pseudo-symmetric submanifold $M^{n}$ in a real space form $\widetilde{M}^{n+m}(\widetilde{c})$ is either minimal or pseudo-umbilical or has flat normal connection, and if the first normal spaces of $M^{n}$ in $\widetilde{M}^{n+m}$ have maximal dimension $n(n+1) / 2$ then $M$ is minimal or pseudo-umbilical in $\widetilde{M}$. All parallel, semi-parallel, and respectively, pseudo-parallel submanifolds $M^{n}$ in real space forms $\widetilde{M}^{n+m}(\widetilde{c})$ are automatically intrinsically locally symmetric, semi-symmetric, and, respectively, pseudosymmetric Riemannian manifolds. For classification results on these submanifolds, a.o. due to Ferus, Dillen and Nölker, Lumiste, Asperti, Lobos, Tojeiro, Mercuri, i.p. see [1, 27, 33, 51, 53].

Recently, the conjecture on the Wintgen inequality, as formulated in [18] was completely resolved in the affirmative by $\mathrm{Lu}$ [52] and by Ge and Tang [34].

Theorem 20 (Lu, Ge and Tang). The Wintgen inequality $\rho \leq H^{2}-\rho^{\perp}+\widetilde{c}$ holds for every submanifold $M^{n}$ in any real space form $\widetilde{M}^{n+m}(\widetilde{c})$, whereby $\rho$ and $\rho^{\perp}$ are the normalised scalar curvature of $M$ and the normalised scalar normal curvature of $M$ in $\widetilde{M}$, respectively, and $H^{2}$ is the squared mean curvature of $M$ in $\widetilde{M}$, and $\rho=H^{2}-\rho^{\perp}+\widetilde{c}$ if and only if, with respect to suitable tangent and normal orthonormal frames $\left\{E_{i}\right\}$ and $\left\{\xi_{\alpha}\right\}$, the shape operators of $M^{n}$ in $\widetilde{M}^{n+m}(\widetilde{c})$ assume the forms

$$
\begin{aligned}
A_{1} & =\left[\begin{array}{ccccc}
\lambda+\mu \cos \theta & 0 & 0 & \cdots & 0 \\
0 & \lambda-\mu \cos \theta & 0 & \cdots & 0 \\
0 & 0 & \lambda & \cdots & 0 \\
\vdots & \vdots & \vdots & \ddots & \vdots \\
0 & 0 & 0 & \cdots & \lambda
\end{array}\right], \quad A_{2}=\left[\begin{array}{ccccc}
\mu \sin \theta & 0 & 0 & \cdots & 0 \\
0 & -\mu \sin \theta & 0 & \cdots & 0 \\
0 & 0 & 0 & \cdots & 0 \\
\vdots & \vdots & \vdots & \ddots & \vdots \\
0 & 0 & 0 & \cdots & 0
\end{array}\right], \\
A_{3} & =\left[\begin{array}{ccccc}
0 & \mu & 0 & \cdots & 0 \\
\mu & 0 & 0 & \cdots & 0 \\
0 & 0 & 0 & \cdots & 0 \\
\vdots & \vdots & \vdots & \ddots & \vdots \\
0 & 0 & 0 & \cdots & 0
\end{array}\right], \quad A_{4}=\cdots=A_{m}=0 .
\end{aligned}
$$

Hereby $\rho=\frac{2}{n(n-1)} \sum_{i<j} R\left(E_{i}, E_{j}, E_{j}, E_{i}\right)$, and $\rho^{\perp}=\frac{2}{n(n-1)}\left[\sum_{i<j} \sum_{\alpha<\beta} R^{\perp}\left(E_{i}, E_{j} ; \xi_{\alpha}, \xi_{\beta}\right)^{2}\right]^{1 / 2}$.

There are many submanifolds satisfying the equality $\rho=H^{2}-\rho^{\perp}+\widetilde{c}$, and, in analogy with Chen's nomenclature for the submanifolds satisfying the equality in several other kinds of general inequalities between intrinsic and extrinsic invariants of submanifolds, these submanifolds are called Wintgen ideal submanifolds. For explicit descriptions of Wintgen ideal submanifolds and for further discussions on this inequality, one can consult amongst others studies by Boruvka [6], Bryant [7, 8], Choi-Lu [12], Dajczer-Tojeiro [13, 14], Dillen-Fastenakels-Van der Veken [29, 30], Eisenhart [31], Guadalupe-Rodriguez [36], Kommerell [45], Rouxel [61] and Wintgen [70]. As was shown in $[24,58,59]$, there are close connections between Wintgen ideal submanifolds and intrinsic pseudo-symmetry conditions. For instance, for $n>3$, every Wintgen ideal submanifold $M^{n}$ in $\widetilde{M}^{n+m}(\widetilde{c})$ has pseudo-symmetric conformal Weyl tensor, and the minimal Wintgen ideal submanifolds are characterised by the fact that $L_{C}=-\frac{n-3}{(n-1)(n-2)} K_{\mathrm{inf}}$; moreover, the Deszcz symmetric Wintgen ideal submanifolds are either totally umbilical, (in particular, then $M$ being 
a real space form), or minimal (in which case $M$ is pseudo-symmetric of constant type, namely $L=\widetilde{c})$.

Finally, we will comment on the fact that the pseudo-symmetric Riemannian manifolds $\left(M^{n}, g\right), n \geq 3$, could well be considered as being the most natural symmetric spaces which, in extension of the perfect symmetry behaviour of the real space forms, do admit privileged directions at all of its points, or, still, as being the most symmetric anisotropic spaces. In particular, for spaces of dimension $n=3$, one has the following.

Theorem 21 ([26]). A 3D Riemannian manifold is Deszcz symmetric if and only if, it is quasi-Einstein.

We recall that $\left(M^{n}, g\right)$ is said to be an Einstein manifold when its Ricci tensor $S$ is proportional to the metric $g$, or, still, when all its Ricci curvatures at all points are equal, and $\left(M^{n}, g\right)$ is said to be quasi-Einstein when at all points it has a Ricci curvature of multiplicity $\geq n-1$; in particular, $\left(M^{n}, g\right)$ is properly quasi-Einstein when at all points it has a Ricci curvature of multiplicity precisely $n-1$ and the other Ricci curvature then of course has multiplicity 1 . And, in this situation, in particular, the Ricci principal direction with respect to this latter Ricci curvature then determines on $\left(M^{n}, g\right)$ a tangent direction which geometrically is essentially different from all other tangent directions. We recall the following.

Theorem 22 (Schouten and Struik). A 3D Riemannian manifold is an Einstein space if and only if it is a real space form.

The real space forms $M^{n}(c)$ are semi-symmetric and thus in particular pseudo-symmetric. So the proper $3 D$ Deszcz symmetric spaces are the proper quasi-Einstein $3 D$ spaces, with a Ricci curvature $\lambda$ of multiplicity 1 and a Ricci curvature $\mu \neq \lambda$ of multiplicity 2 , and $L=\lambda / 2$ : $M \rightarrow \mathbb{R}$. In his approach to geometries as topological manifolds $M$ endowed with transformation groups $G$ satisfying by definition just a few conditions such as to allow for the class of real space forms to be enlarged so as to moreover incorporate certain still homogeneous, but essentially anisotropic spaces, Thurston [65, 66] introduced his so-called model geometries which can be seen as to determine successfully some of the most natural anisotropic geometrical manifolds $\left(M^{n}, G\right), n \geq 3$, beyond the projective class of the Euclidean and the classical non-Euclidean geometries. The introduction of the pseudo-symmetric spaces, i.e. of the projective class of the semi-symmetric spaces, is a metrical approach also to determine some of the most natural anisotropic manifolds through the symmetry property given by the invariance of the curvature of the connection $\nabla$ on a differential manifold $M$ under the parallel transport corresponding to $\nabla$ around the infinitesimal co-ordinate parallelograms on $M$. We recall that the eight $3 D$ Thurston model geometries are $\mathbb{E}^{3}, \mathbb{S}^{3}, \mathbb{H}^{3}, \mathbb{S}^{2} \times \mathbb{E}^{1}, \mathbb{H}^{2} \times \mathbb{E}^{1}$, the Heisenberg group $H_{3}$, the covering group of the special linear group $\widetilde{S L}(2, \mathbb{R})$ and the solvable Lie group Sol. A canonical metric can be placed on each of the model spaces, which, except for $\mathbb{H}^{3}$ and $S o l$, can be written as $\mathrm{d} s^{2}=\frac{d x^{2}+d y^{2}}{\left[1+m\left(x^{2}+y^{2}\right)\right]^{2}}+\left[d z+\frac{l}{2} \frac{y d x-x d y}{1+m\left(x^{2}+y^{2}\right)}\right]^{2}$, whereby if $m=l=0, M=\mathbb{E}^{3} ; 4 m-l^{2}=0$, $M=\mathbb{S}^{3}$; if $m<0$ and $l=0, M=\mathbb{H}^{2} \times \mathbb{E}^{1}$; if $m>0$ and $l=0, M=\mathbb{S}^{2} \times \mathbb{E}^{1}$; if $m<0$ and $l \neq 0, M=\widetilde{S L}(2, \mathbb{R})$; and if $m=0$ and $l \neq 0, M=H_{3}$. On the Lie group Sol, one can put the metric $\mathrm{d} s^{2}=\mathrm{e}^{2 z} \mathrm{~d} x^{2}+\mathrm{e}^{-2 z} \mathrm{~d} y^{2}+\mathrm{d} z^{2}$. Besides the real space forms $\mathbb{E}^{3}, \mathbb{S}^{3}$ and $\mathbb{H}^{3}$, the five other geometries are proper quasi-Einstein, and so one has the following.

Theorem 23 ([2]). All 3D Thurston geometries are either spaces of constant curvature or Deszcz symmetric of constant type; (for $\mathbb{E}^{3}, \mathbb{S}^{3}$ and $\mathbb{H}^{3}, K=0,1$ and -1 respectively; for $\mathbb{S}^{2} \times \mathbb{E}^{1}$ and $\mathbb{H}^{2} \times \mathbb{E}^{1}, L=0 ;$ for $H_{3}$ and $\widetilde{S L}(2, \mathbb{R}), L=1$; and for Sol, $\left.L=-1\right)$.

Deleting Sol from the $3 D$ Thurston spaces and adding instead $S U(2)$, with the above metric whereby $m>0$ and $l \neq 0$, one obtains the list of the $3 D$ so-called d'Atri spaces, i.e. the 
Riemannian manifolds for which locally all geodesic reflections are volume preserving. One has the following.

Theorem 24 ([2]). All 3D d'Atri spaces are Deszcz symmetric of constant type (besides the above, for $S U(2), L=1)$.

Basically, Theorem 21 readily follows from the fact that for every Riemannian manifold of dimension 3 the Weyl conformal curvature tensor $C$ automatically vanishes. In the above respects, for higher dimensions one has the following.

Theorem 25 ([17, 21]). A conformally flat Riemannian manifold $\left(M^{n}, g\right)$ of dimension $n \geq 4$ is Deszcz symmetric if and only if it has at most two distinct Ricci curvatures (of arbitrary multiplicities).

For the Einstein spaces $S=\lambda g$ this again actually only concerns the real space forms, and in case the spaces under consideration are not Einstein, the two orthogonally complementary eigenspaces of their Ricci tensor consist of geometrically non-equivalent tangent directions, thus realising on these pseudo-symmetric spaces a manifest, yet in some sense elementary, anisotropy.

As was already mentioned in the Introduction, the above essentially goes through for semiRiemannian spaces. In particular, in [37] a classification, based on the algebraic properties of the Weyl and Ricci tensor, was obtained for the 4-dimensional pseudo-symmetric space-times. It follows that most of the well-known space-times, such as the Schwarzschild metric, the ReissnerNördstrom metric, the Kottler metric and the Friedmann-Lemaitre-Robertson-Walker metrics, are pseudo-symmetric but not semi-symmetric. Moreover, the first three metrics are examples of non-conformally flat, pseudo-symmetric spaces. See also [16, 25] for more details.

\section{Acknowledgements}

The authors do thank the referees whose comments resulted in real improvements of the original version of this paper. The first author was partially supported by the Spanish MEC Grant MTM2007-60731 with FEDER funds and the Junta de Andalucía Regional Grant P06-FQM01951. Both authors were partially supported by the Research Foundation Flanders project G.0432.07.

\section{References}

[1] Asperti A., Lobos G., Mercuri F., Pseudo-parallel submanifolds of a space-form, Adv. Geom. 2 (2002), $57-71$.

[2] Belkhelfa M., Deszcz R., Verstraelen L., Symmetry properties of 3-dimensional d'Atri spaces, Kyungpook Math. J. 46 (2006), 367-376.

[3] Berger M., La géométrie métrique des variétés riemanniennes (variations sur la formule $a^{2}=b^{2}+c^{2}-$ $2 b c \cos \alpha$ ), in Élie Cartan et les mathématiques d'aujourd'hui, Astérisque, Paris, 1985, 9-66.

[4] Berger M., A panoramic view of Riemannian geometry, Springer-Verlag, Berlin, 2003.

[5] Boeckx E., Foliated semi-symmetric spaces, Ph.D. Thesis, K.U. Leuven, Leuven, Belgium, 1995.

[6] Boruvka O., Sur une classe de surfaces minima plongées dans un espace à quatre dimensions à courbure constante, Bull. Int. Česka Acad. Praze 29 (1928), 256-277.

[7] Bryant R.L., Some remarks on the geometry of austere manifolds, Bol. Soc. Brasil. Math. (N.S.) 21 (1991), 133-157.

[8] Bryant R.L., Second order families of special Lagrangian 3-folds, in Perspectives in Riemannian Geometry, CRM Proc. Lecture Notes, Vol. 40, Amer. Math. Soc., Providence, RI, 2006, 63-98, math.DG/0007128.

[9] Cecil T., Lie sphere geometry, with applications to submanifold, 2nd ed., Springer-Verlag, New-York, 2007.

[10] Cecil T., Isoparametric and Dupin hypersurfaces, SIGMA 4 (2008), 062, 28 pages, arXiv:0809.1433. 
[11] Chen B.-Y., Totally umbilical submanifolds, Soochow Math. J. 5 (1980), 9-37.

[12] Choi T., Lu Z., On the DDVV conjecture and the comass in calibrated geometry. I, Math. Z. 260 (2008), 409-429, math.DG/0610709.

[13] Dajczer M., Tojeiro R., Submanifolds of codimension two attaining equality in an extrinsic inequality, Math. Proc. Cambridge Philos. Soc. 146 (2009), 461-474, arXiv:0802.0805.

[14] Dajczer M., Tojeiro R., All superconformal surfaces in $\mathbb{R}^{4}$ in terms of minimal surfaces, Math. Z. 261 (2009), 869-890, arXiv:0710.5317.

[15] Defever F., Deszcz R., A note on geodesic mappings of pseudosymmetric Riemannian manifolds, Colloq. Math. 62 (1991), 313-319.

[16] Defever F., Deszcz R., Verstraelen L., Vrancken L., On pseudosymmetric space-times, J. Math. Phys. 35 (1994), 5908-5921.

[17] Deprez J., Deszcz R., Verstraelen L., Examples of pseudo-symmetric conformally flat warped products, Chinese J. Math. 17 (1989), 51-65.

[18] De Smet P.J., Dillen F., Verstraelen L., Vrancken L., A pointwise inequality in submanifold theory, Arch. Math. (Brno) 35 (1999), 115-128.

[19] Deszcz R., On pseudosymmetric spaces, Bull. Soc. Math. Belg. Sér. A 44 (1992), 1-34.

[20] Deszcz R., Grycak W., On some class of warped product manifolds, Bull. Inst. Math. Acad. Sinica 15 (1987), 311-322.

[21] Deszcz R., Grycak W., On certain curvature conditions on Riemannian manifolds, Colloq. Math. 58 (1990), 259-268.

[22] Deszcz R., Haesen S., Verstraelen L., Classification of space-times satisfying some pseudo-symmetry type conditions, Soochow J. Math. 30 (2004), 339-349.

[23] Deszcz R., Haesen S., Verstraelen L., On natural symmetries, Chapter 6 in "Topics in Differential Geometry", Editors A. Mihai, I. Mihai and R. Miron, Editura Academiei Române, 2008.

[24] Deszcz R., Petrović-Torgašev M., Șentürk Z., Verstraelen L., Characterisation of the pseudo-symmetries of ideal Wintgen submanifolds of dimension 3, Preprint, 2007.

[25] Deszcz R., Verstraelen L., Vrancken L., The symmetry of warped product spacetimes, Gen. Relativity Gravitation 23 (1991), 671-681.

[26] Deszcz R., Verstraelen L., Yaprak Ș., Warped products realizing a certain condition of pseudosymmetry type imposed on the Weyl curvature tensor, Chinese J. Math. 22 (1994), 139-157.

[27] Dillen F., Nölker S., Semi-parallelity, multi-rotation surfaces and the helix property, J. Reine Angew. Math. 435 (1993), 33-63.

[28] Dillen F., Fastenakels J., Haesen S., Van der Veken J., Verstraelen L., Submanifold theory and the parallel transport of Levi-Civita, Preprint.

[29] Dillen F., Fastenakels J., Van der Veken J., A pinching theorem for the normal scalar curvature of invariant submanifolds, J. Geom. Phys. 57 (2007), 833-840.

[30] Dillen F., Fastenakels J., Van der Veken J., Remarks on an inequality involving the normal scalar curvature, in Pure and Applied Differential Geometry - PADGE 2007, Shaker Verlag, Aachen, 2007, 83-92, math.DG/0610721.

[31] Eisenhart L.P., Minimal surfaces in Euclidean four space, Am. J. Math. 34 (1912), 215-236.

[32] Eisenhart L.P., Riemannian geometry, Princeton University Press, Princeton, NJ, 1997.

[33] Ferus D., Symmetric submanifolds of Euclidean spaces, Math. Ann. 247 (1980), 81-93.

[34] Ge J., Tang Z., A proof of the DDVV conjecture and its equality case, Pacific J. Math 237 (2008), 87-95, arXiv:0801.0650.

[35] Grycak W., On semi-decomposable 2-recurrent Riemannian spaces, Sci. Papers Inst. Math. Wroctaw Techn. Univ. 16 (1976), 15-25.

[36] Guadalupe I.V., Rodriguez L., Normal curvature of surfaces in space forms, Pacific J. Math. 106 (1983), 95-103.

[37] Haesen S., Verstraelen L., Classification of the pseudosymmetric space-times, J. Math. Phys. 45 (2004), 2343-2346. 
[38] Haesen S., Verstraelen L., Curvature and symmetries of parallel transport, Chapter 8 in "Differential Geometry and Topology, Discrete and Computational Geometry", Editors M. Boucetta and J.-M. Morvan, NATO Science Series III, Vol. 197, IOS Press, 2005.

[39] Haesen S., Verstraelen L., Properties of a scalar curvature invariant depending on two planes, Manuscripta Math. 122 (2007), 59-72.

[40] von Helmholtz H., Über die Thatsachen, die der Geometrie zum Grunde liegen, in Über Geometrie, Wissenschaftliche Buchgesellschaft, Darmstadt, 1968.

[41] Jahanara B., Haesen S., Șentürk Z., Verstraelen L., On the parallel transport of the Ricci curvatures, J. Geom. Phys. 57 (2007), 1771-1777.

[42] Jahanara B., Haesen S., Petrović-Torgašev M., Verstraelen L., On the Weyl curvature of Deszcz, Publ. Math. Debrecen 74 (2009), 417-431.

[43] Kobayashi S., Nomizu K., Foundations of differential geometry, Vol. I, John Wiley and Sons, New York, 1963.

[44] Kobayashi S., Nomizu K., Foundations of differential geometry, Vol. II, John Wiley and Sons, New York, 1969.

[45] Kommerell K., Riemann'sche Flächen in ebenen Raum von vier Dimensionen, Math. Ann. 60 (1905), 548596.

[46] Kowalski O., An explicit classification of 3-dimensional Riemannian spaces satisfying $R(X, Y) \cdot R=0$, Czechoslovak Math. J. 46 (1996), 427-474.

[47] Kowalski O., Sekizawa M., Pseudo-symmetric spaces of constant type in dimension three - elliptic spaces, Rend. Mat. Appl. (7) 17 (1997), 477-512.

[48] Kowalski O., Sekizawa M., Pseudo-symmetric spaces of constant type in dimension three - non-elliptic spaces, Bull. Tokyo Gakugei Univ. (4) 50 (1998), 1-28.

[49] Kühnel W., Differential geometry. Curves - surfaces - manifolds, 2nd ed., Student Mathematical Library, Vol. 16, American Mathematical Society, Providence, RI, 2006.

[50] Levi-Civita T., Nozione di parallelismo in una varietà qualunque e conseguente specificazione geometrica della curvatura Riemanniana, Rend. Cir. Mat. Palermo 42 (1917), 173-205.

[51] Lobos G.A., Tojeiro R., Pseudo-parallel submanifolds with flat normal bundle of space forms, Glasg. Math. J. 48 (2006), 171-177.

[52] Lu Z., On the DDVV conjecture and the comass in calibrated geometry. II, arXiv:0708.2921.

[53] Lumiste Ü., Semiparallel submanifolds in real space forms, Springer, New York, 2009.

[54] Matveev V.S., Geometric explanantion of the Beltrami theorem, Int. J. Geom. Methods Mod. Phys. 3 (2006), 623-629.

[55] Mikeš J., Geodesic mappings of special Riemannian spaces, in Topics in Differential Geometry (Hajduszoboszló 1984), Colloq. Math. Soc. János Bolyai, Vol. 46, North-Holland, Amsterdam, Vol. II, 1988, 793-813.

[56] Nomizu K., On hypersurfaces satisfying a certain curvature condition on the curvature tensor, Tôhoku Math. J. (2) 20 (1968), 46-59.

[57] Olszak Z., On totally umbilical submanifolds of Cartan symmetric manifolds, Demonstratio Math. 9 (1976), 95-103.

[58] Petrović-Torgašev M., Verstraelen L., On Deszcz symmetries of Wintgen ideal submanifolds, Arch. Math. (Brno) 44 (2008), 57-67.

[59] Petrović-Torgašev M., Verstraelen L., Intrinsic curvature properties of Wintgen ideal submanifolds, Preprint, 2007.

[60] Riemann B., Über die Hypothesen, welche der Geometrie zu Grunde liegen, in Gaußsche Flächentheorie, Riemannsche Räume und Minkowski-Welt, Teubner Verlagsgesellschaft, Leipzig, 1984.

[61] Rouxel B., Sur une famille de A-surfaces d'un espace euclidien $\mathbb{E}^{4}$, in Proc. X Österreichischer Mathematiker Kongress, Innsbrück, 1981, 185.

[62] Schouten J.A., Die direkte Analysis zur neueren Relativitätstheorie, Verhan. Konink. Akad. Wet. Amsterdam 12 (1918), no. 6, 1-95.

[63] Szabó Z.I., Structure theorems on Riemannian spaces satisfying $R(X, Y) \cdot R=0$. I. The local version, J. Differential Geom. 17 (1982), 531-582. 
[64] Szabó Z.I., Structure theorems on Riemannian spaces satisfying $R(X, Y) \cdot R=0$. II. Global version, Geom. Dedicata 19 (1985), 65-108.

[65] Thurston W.P., Three-dimensional manifolds, Kleinian groups and hyperbolic geometry, Bull. Amer. Math. Soc. (N.S.) 6 (1982), 357-381.

[66] Thurston W.P., Three-dimensional geometry and topology, Vol. 1, Princeton Mathematical Series, Vol. 35, Princeton University Press, Princeton, NJ, 1997.

[67] Venzi P., On geodesic mappings in Riemannian or pseudo-Riemannian manifolds, Tensor (N.S.) 32 (1978), 193-198.

[68] Verstraelen L., Comments on pseudo-symmetry in the sense of Ryszard Deszcz, in "Geometry and Topology Of Submanifolds, VI", Editors F. Dillen et al., World Sci. Publ., River Edge, NJ, 1994, 199-209.

[69] Weyl H., Symmetry, Princeton University Press, Princeton, N.J., 1952.

[70] Wintgen P., Sur l'inégalité de Chen-Willmore, C. R. Acad. Sci. Paris Sér. A-B 288 (1979), A993-A995.

[71] Wolf J.A., Spaces of constant curvature, MacGraw-Hill, New York, 1967. 